\section{RELATO DE CASO}

\section{ABDOME AGUDO OBSTRUTIVO POR DIVERTÍCULO DE MECKEL EM ADULTO

\author{
OBSTRUCTIVE ACUTE ABDOMEN BY MECKEL'S DIVERTICLE IN \\ ADULT
}

Stéphanie Gonçalves de Oliveira', Bárbara Chaves Lopes Machado², Pedro Manuel Gonzalez Cuellar ${ }^{3}$, Fellipe Camargo Ferreira Dias ${ }^{4}$, Virgílio Ribeiro Guedes 5 .

\section{RESUMO}

O Divertículo de Meckel é a anomalia congênita mais encontrada no intestino delgado. A alteração é causada pela obliteração incompleta do ducto onfalomesentérico. Geralmente a doença diverticular é assintomática e a probabilidade de manifestar sintomas, como obstrução intestinal, diminui com a idade. Embora à maioria permaneça em silêncio, é importante sempre considerar o divertículo de Meckel como um diagnóstico diferencial em todos os pacientes que apresentem abdome agudo para evitar morbidade e mortalidade associada a complicações tardias.

Palavras-chave: divertículo de Meckel; obstrução intestinal; abdome agudo obstrutivo.

Citação: Oliveira SG, Machado BCL, Pedro Cuellar PMG, Dias FCF, Guedes VR (2018) Abdome agudo obstrutivo por divertículo de Meckel em adulto. Revista de Patologia do Tocantins, 5(4): 44-46.

Instituição: ${ }^{1}$ Acadêmico(a) de Medicina da Universidade Federal do Tocantins, Tocantins, Brasil; ${ }^{2}$ Cirurgiã-geral Universidade Federal do Tocantins/ Hospital Geral de Palmas; ${ }^{3}$ Docente, Cirurgião Geral, Universidade Federal do Tocantins, Tocantins, Brasil; ${ }^{4}$ Médico, Mestrando em Ensino em Ciências e Saúde, Universidade Federal do Tocantins, Tocantins, Brasil; ${ }^{5}$ Docente, Médico Patologista, Universidade Federal do Tocantins, Tocantins, Brasil.

\section{Autor correspondente:}

Stéphanie Gonçalves de Oliveira;

stephaniie846@gmail.com

Editor: Guedes V. R. Medicina, Universidade Federal do Tocantins, Brasil.

Publicado: 09 de dezembro de 2018.

Direitos Autorais: (c) 2018 Oliveira et al. Este é um artigo de acesso aberto que permite o uso, a distribuição e a reprodução sem restrições em qualquer meio, desde que o autor original e a fonte sejam creditados.

Conflito de interesses: os autores declararam que não existem conflitos de interesses.

\section{ABSTRACT}

The Meckel Diverticulum is the most common congenital anomaly found in the small intestine. The change is caused by incomplete obliteration of the omphalomesenteric duct. Diverticular disease is usually asymptomatic and the likelihood of manifesting symptoms, such as bowel obstruction, decreases with age. Although most remain silent, it is important to always consider Meckel's diverticulum as a differential diagnosis in all patients with acute abdomen to avoid morbidity and mortality associated with late complications. The following report is about a rare case of obstructive acute abdome in adult patients caused by the presence of a meckel diverticulo.

Keywords: meckel diverticulum; intestinal obstruction; obstructive acute abdomen. 


\section{INTRODUÇÃO}

O Divertículo de Meckel é a anomalia congênita mais comumente encontrada no intestino delgado. Trata-se de uma projeção intestinal que envolve todas as suas camadas'. São conhecidos pela regra dos dois: incidem em $2 \%$ da população ${ }^{2}$, apenas $2 \%$ são descobertos antes dos dois anos de idade $(53 \%)^{3}$, seu comprimento e diâmetro normalmente não excedem 2 polegadas ( 5 $\mathrm{cm})$, são localizados a 2 pés $(60 \mathrm{~cm})$ da válvula ileocecal ${ }^{2}$ e a relação de incidência entre homens e mulheres é de $2: 1^{3}$. Essa alteração foi identificada pela primeira vez em 1598 por Fadricius Hidanus, mas só foi descrito em detalhes patológicos e embriológicos em 1809 por Jonhan Meckel 4.

A anomalia congênita é causada pela obliteração incompleta do ducto onfalomesentérico durante a oitava semana de gestação ${ }^{3}$. Durante o desenvolvimento embrionário, o saco vitelínico se conecta ao intestino primitivo através do ducto onfalomesentérico ${ }^{2}$. A falha na obliteração resulta no divertículo de Meckel, que se apresenta desde um simples abaulamento, podendo passar despercebido, a uma grande projeção do intestino que se comunica com o umbigo através de um cordão fibroso ou fístula'.

\section{RELATO DE CASO}

D.F.L, 21 anos, masculino, solteiro, natural e procedente de Miracema do Tocantins, deu entrada no serviço de emergência do Hospital Geral de Palmas no dia 18/02/2018. Na ocasião, o paciente apresentava dor abdominal há 5 dias, tipo cólica, difusa, com piora progressiva, mais intensa em epigástrio, associada a parada de eliminação de flatos e fezes, náuseas e vômitos. Negava febre e alterações urinárias. Ao exame físico apresentava bom estado geral, lúcido e orientado em tempo e espaço, febril, acianótico e anictérico, hipocorado (+/+4), hidratado, PA: 149/94, pulso $110 \mathrm{bpm}$, saturação de 02. 95\%. O abdome apresentava ruídos hidroaéreos aumentados, distendido, com dor a palpação difusa e sinais de irritação peritoneal em todos os quadrantes. Ao toque retal havia presença de pequena quantidade de fezes em ampola retal.

Solicitado radiografia de abdome que revelou presença de níveis hidroaéreos em ampola retal, sem presença de pneumoperitônio. Levantado hipótese diagnóstica de abdome agudo obstrutivo foi indicado tratamento cirúrgico por laparotomia exploradora. Durante o procedimento cirúrgico foi identificado moderada quantidade de líquido livre turvo em cavidade abdominal, presença de obstrução em íleo a $50 \mathrm{~cm}$ da válvula ileocecal causada por pinçamento da alça por divertículo de Meckel, o qual se encontrava necrosado em toda sua extensão (Figura 1 e 2), exceto em sua base, além de aderência fixa entre alça de delgado e parede anterior do reto, não sendo identificadas outras lesões em outros órgãos. Realizado diverticulectomia seguida de enterorrafia no local (base) (Figura 3).

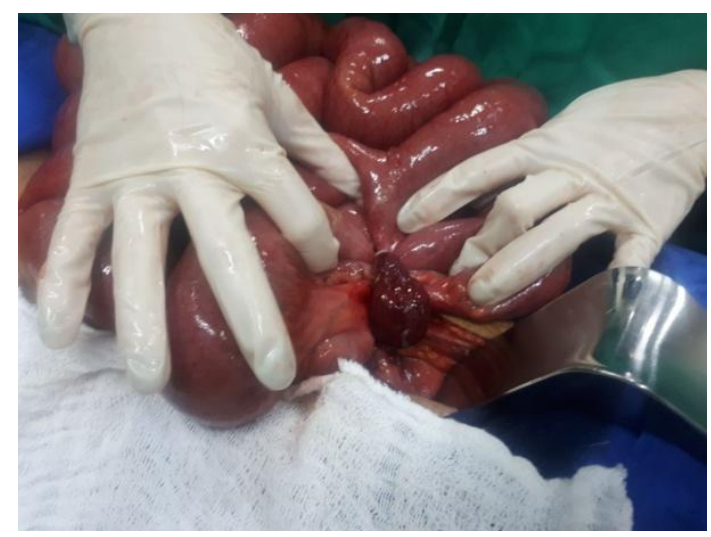

Figura 1
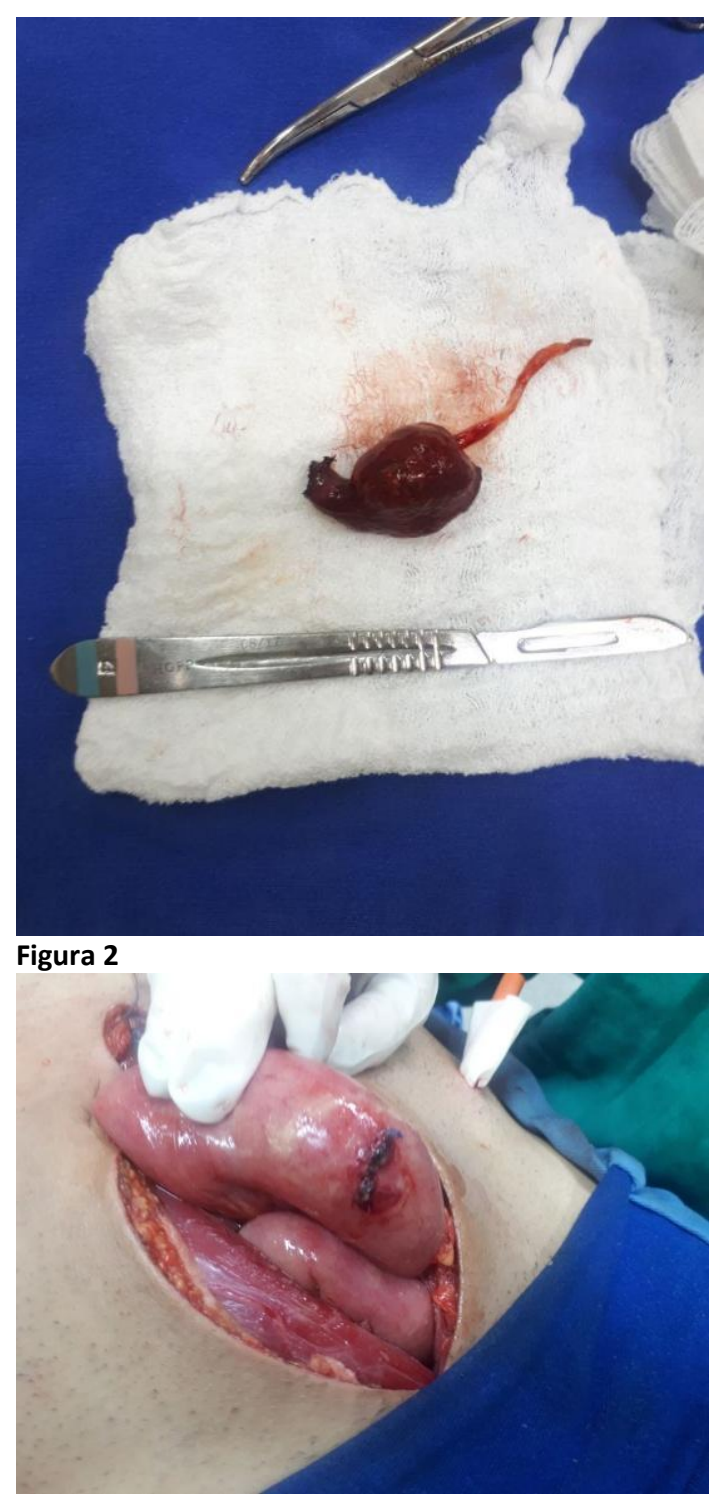

Figura 3

No quinto dia de pós operatório o paciente apresentou fístula entérica. Foi então iniciada dieta parenteral e suspendida dieta via oral. Paciente apresentou melhora do débito da fístula no nono dia pós operatório, sendo então retirado os drenos abdominais e indicado alta hospitalar com acompanhamento em ambulatório. $\mathrm{O}$ anátomo patológico revelou presença de enterite crônica intensa em atividade acentuada, porém, não foi descrito presença de tecido ectópico.

\section{DISCUSSÃO}

A doença diverticular geralmente é assintomática, mas, em alguns pacientes, podem manifestar com variadas apresentações clínicas, como diverticulite, hemorragia retal e obstrução intestinal ${ }^{5}$. Grande parte dos casos de DM são encontrados incidentalmente, durante o intra operatório por outro motivo ${ }^{4}$.

Em crianças a manifestação mais comum é a hemorragia digestiva ${ }^{2}$. Recentemente, foi publicado um estudo retrospectivo pela Universidade de Medicina de Wenzhou na China envolvendo pacientes pediátricos de até 14 anos. No período de 2001 a 2015, eles registraram no seu instituto, 102 casos de DM, dos quais 65 eram meninos e 37 meninas. 0 estudo revela que 41 manifestaram hemorragia digestiva baixa, 32 intussuscepção, 12 peritonite, 8 perfuração de DM, 4 diverticulite, 7 obstrução intestinal e 10 foram achados incidentais intra operatórios por outro motivo6. 
A probabilidade da doença se tornar sintomática diminui com a idade ${ }^{3}$. Em adultos, os DM se manifestam principalmente como obstrução intestinal, como no caso relatado, seguida de intussuscepção, ulcerações, fístulas e tumores ${ }^{2}$. A perfuração é uma complicação rara e pode ter manifestações semelhantes à apendicite aguda7.

As células que constituem o ducto onfalomesentérico são pluripotentes, o que explica a presença de tecido heterotópico em alguns casos. Células da mucosa gástrica estão presentes em $50 \%$ dos divertículos e são fonte de úlcera crônica induzida pelo $\mathrm{HCl}$ no tecido intestinal adjacente. A presença de tecido pancreático, presente em $5 \%$ dos casos, pode levar à inflamação, simulando pancreatite aguda com elevação de amilase'.

Ultrassonografia e a tomografia computadorizada não são bons exames para diagnóstico pré operatório do divertículo de Meckel, pois não são capazes de diferenciar uma alça intestinal de um divertículo. O melhor exame é a cintilografia com technet $99 \mathrm{mTc}$, capaz de detectar não só a presença do divertículo, mas também a presença de mucosa heterotópica ou locais de sangramento gastrointestinal ${ }^{4}$. Existem relatos na literatura de casos em que o diagnóstico do divertículo de Meckel foi realizado usando tomografia computadorizada, ressonância magnética, cápsula enteroscópica ou enteroscopia com balão duplo ${ }^{8}$.

\section{CONCLUSÃO}

A obstrução intestinal por Divertículo de Meckel em adultos é uma causa rara de abdome agudo. Embora esta anomalia esteja presente em apenas $2 \%$ da população e a maioria permanece assintomática, é importante sempre considerar o Divertículo de Meckel como um diagnóstico diferencial em todos os pacientes que apresentem abdome agudo, para evitar morbidade e mortalidade associada a complicações tardias.

\section{BIBLIOGRAFIA}

1. Sabiston. Tratado de cirurgia: A base biológica da prática cirúrgica moderna. 19.ed. Saunders. Elsevier.

2. Darlington CD., Anitha GFS. Meckel's diverticulitis masquerading as acute pancreatitis: a diagnostic dilema. Indian Journal of Critical Care Medicine 2017, november 21. Available from

https://www.ncbi.nlm.nih.gov/pmc/articles/PMC5699010/

3. Capelão SM, Hilário S, Laureano $\mathrm{M}$, Nobre J, Gonçalves I. Intestinal obstructions by giant Meckel's diverticulum. Ge Portgastroenterol 2017. Available from : https://www.ncbi.nlm.nih.gov/pmc/articles/PMC5729947/

4. Georges A, Hager A. Case report: preoperatively diagnosed perforated Meckel's diverticulum containing gastric and pancreatic-type mucosa. BioMedCentral 2017. Available from : https://www.ncbi.nlm.nih.gov/pmc/articles/PMC5387318/

5. Nastos C., Giamnoulopoulos, Georgopoulos, Salakos C., Dellaportas D., Papaconstantinou I, Theodousopoulos T., Polynemeas G. Large enterolith complicating a Meckel diverticulum causing ileus in an adolescent male patient. Case Reports in Surgery, 2017 december 17. Available from : https://www.ncbi.nlm.nih.gov/pmc/articles/PMC5748096/

6. Xiao-Kun L, Xiao-zho N, Xiao-zhou B, Na Z, Quiong-zhang X, Cong-de $C$ Clinical characteristics of Meckel diverticulum in children A retrospective review of a 15-year single-center experience. Medicine Baltimore, 2017 august, 11. Available from https://www.ncbi.nlm.nih.gov/pmc/articles/PMC5556236/

7. Liu K, Wu Y. Spontaneous perforation of Meckel diverticulum: A case report. Medicine, 2017 december. Available from: https://journals.lww.com/md-
journal/Fulltext/2017/12290/Spontaneous perforation of Me ckel diverticulum A.60.aspx

8. Pietrzak J, Obuchowicz A, Majda D, Kiedos A. Meckel's diverticulum - a congenital defect of the gastrointestinal tract underestimated in differential diagnostics. Own experience. Developmental Period Medicine 2017. Available from: http://medwiekurozwoj.pl/articles/2017-1-5.pdf 